\title{
Chapter 7-Summary
}

This chapter illustrates some of the clinical consequences of defective neural connections and/or myogenic responses in the gut. The vagueness of the clinical definitions or descriptions for various disorders and syndromes bear testament to the fact that their aetiology is poorly understood. It is becoming increasingly apparent that these conditions are multifactorial and that central as well as peripheral sites are involved to varying degrees. The autonomic nervous system serves as the important link between the enteric nervous system and the central nervous system (CNS) and may be open to pharmacological manipulation in certain bowel disorders. While autonomic function generally maintains homeostasis, over activity of any of its elements may lead to over reaction from the CNS, and an exaggerated behavioural as well as physiological response. The development of new techniques which assess different aspects of autonomic function should hopefully aid in the search for autonomic "defects" in certain functional disorders. However, it is apparent that the criteria necessary to define and diagnose enteric disorders are poorly established, and where these conditions relate to abnormal numbers or abnormal differentiation of neurones, they are generally only recognised consistently if they are at the severe end of the spectrum, for example aganglionosis in
Hirschsprung's disease. In this chapter, the limitations of the experimental methods available for pathological definition of autonomic defects are weighed against the inadequacies of clinical definitions which give an "overview" of the condition but do not allow the root of the problem to be identified and targeted with treatment. At the same time, the dilemma of the gastroenterologist or gastrointestinal surgeon who is faced with the "end product" of the disorder (for example, postoperative ileus) and may have to deal with it urgently, has been addressed. It is clear that we require extensive research into not only mechanisms but also suitable models (including human ones) for studying possible central or peripheral nervous defects in functional gastrointestinal disorders. It has been suggested that by studying the "end product" (for example, intestinal pseudo-obstruction or postoperative ileus) we may be able to work back towards a solution, using pharmacological tools.

In conclusion, this chapter has highlighted the need to link experience in research methodology with clinical experience in order to improve our understanding of the mechanisms underlying some relatively common gastrointestinal disorders. 